\title{
Investigação da memória autobiográfica em idosos com Demência de Alzheimer nas fases leve e moderada
}

\author{
Caroline Araújo Lemos \\ Universidade Potiguar \\ Izabel Hazin \\ Jorge Tarcísio da Rocha Falcão \\ Universidade Federal do Rio Grande do Norte
}

\begin{abstract}
Resumo
O presente trabalho apresenta dados de pesquisa referentes à investigação de memória autobiográfica (MA) em idosos com Demência de Alzheimer (DA) nas fases leve e moderada. Participaram do estudo quarenta e quatro idosos, divididos em três grupos: DA leve $(n=15)$ e DA moderada $(n=15)$; e 14 idosos sem histórico de alterações neuropsiquiátricas que constituíram o grupo Controle. Utilizou-se na avaliação da memória autobiográfica versão reduzida do Teste de Memória Autobiográfica (TMA) e do Questionário de Memória Autobiográfica (QMA). Os dados evidenciaram diferenças significativas entre os grupos representantes da variável independente (estados leve e moderado de DA) e o grupo Controle, tendo-se verificado neste grupo maior número de memórias específicas, com elevada intensidade vivencial das características fenomenais da recordação, quando comparado aos grupos com DA. Tais dados permitem concluir que alterações na MA em sujeitos com DA podem ser observadas desde a fase inicial da doença, tanto no que diz respeito à capacidade de especificar a recordação, quanto com relação às características fenomenais da lembrança.
\end{abstract}

Palavras-chave: Demência de Alzheimer; memória autobiográfica; teste de memória autobiográfica; questionário de memória autobiográfica.

\begin{abstract}
Autobiographical memory investigation among aged Alzheimer's disease patients in mild and moderate stages. This research investigated autobiographical memory (AM) in elderly individuals having Alzheimer's disease (AD) in mild and moderate stages. 44 aged persons participated in this research, divided in three sub-samples: AD in mild stage $(n=15)$, AD in moderate stage $(n=15)$ and subjects without morbid indication of AD (control group). A short version of Autobiographical Memory Test (AMT) and Autobiographical Memory Questionnaire were used for memory evaluation. Data showed significant differences between AD (mild and moderate) and Control groups, the former being able to recall a greater number of specific memories, along with elevated emotional intensity in phenomenal qualities of these memories, when compared to the other two AD groups. These findings allow the main conclusion that changes in autobiographic memory among AD patients can be observed since early stages of this disease, in terms of both capacity of recalling and emotional quality of remembered content.
\end{abstract}

Keywords: Alzheimer's Disease; autobiographical memory; autobiographical memory test; autobiographical memory questionnaire.

A necessidade de investigações acerca do processo de envelhecimento em diferentes domínios tem aumentado nos dias atuais, uma vez que a expectativa de vida cresceu em praticamente todo o mundo. Dentre os problemas de saúde que ocorrem com maior frequência nesta fase de vida está a demência, cujo número de novos casos aumenta com a progressão da idade (Scazufca et al., 2008), sendo a Demência de Alzheimer (DA) a mais comum no idoso- cerca de 50 a $60 \%$ dos casos - com apresentação clínica e patológica bem definida, ainda que o diagnóstico definitivo seja necessariamente dado post mortem (Herrera, Caramelli, Silveira, \& Nitrini, 2002; Machado, 2002). A sintomatologia da DA pode ser dividida em três estágios (leve, moderada e avançada), ressaltando-se que a hierarquia da progressão dos sintomas na descrição do curso clínico da doença pode sofrer alterações individuais, dependendo de variáveis, tais como a idade de início de sintoma, o sexo e o nível educacional (Machado, 2002).

A taxa de incidência da DA aumenta quase exponencialmente com o passar dos anos até os 85 anos de idade (Qiu, Kivilpelto, \& Strauss, 2009), atingindo valores de prevalência superiores a $50 \%$ em indivíduos com 95 anos ou mais (Vilela \& Caramelli, 2006). No Brasil, os dados epidemiológicos são semelhantes aos encontrados no restante do mundo, com a taxa de incidência de 7,7 por 1000 pessoas ao ano desenvolvendo DA entre as 
pessoas com idade superior a 65 anos (Qiu et al., 2009; Vilela \& Caramelli, 2006). Tal constatação aponta para a necessidade de pesquisas voltadas para a oferta de subsídios que auxiliem no diagnóstico precoce dessa doença, visando a prevenção de agravos e maior efetividade no tratamento.

Segundo a definição do DSM-IV-TR (American Psychiatric Association, 2002), os quadros demenciais são caracterizados pela presença de déficits cognitivos múltiplos, adquiridos e persistentes, dentre os quais se destaca o déficit de memória, acompanhado de outra alteração cognitiva (afasia e/ou apraxia e/ ou agnosia e/ou disfunção executiva). Tais alterações devem ser suficientes para causar comprometimento das atividades sócioocupacionais e representar declínio significativo em relação ao conjunto de habilidades cognitivas que caracterizavam o sujeito antes do diagnóstico da doença.

$\mathrm{Na} \mathrm{DA}$, a memória é a função cognitiva mais precocemente prejudicada, estando tal déficit parcialmente associado ao declínio progressivo e intenso do número e eficiência dos neurônios. No que diz respeito aos déficits de memória comuns na DA, verificase já nas fases iniciais da doença o comprometimento da memória episódica de longo prazo e da memória de curto prazo, estando a intensidade de tais déficits relacionados à gravidade do quadro (Abreu, Forlenza, \& Barros, 2005). Nesse sentido, o impacto da doença sobre a memória autobiográfica (MA) merece destaque.

Para Greenberg e Rubin (2003), a Memória Autobiográfica se caracteriza por uma série de propriedades específicas que justificam sua classificação como sistema específico independente. Inicialmente, como memória episódica, armazena informações autobiográficas sobre datas de episódios ou eventos e relações espaço-temporais entre eles. Entretanto, tais memórias ultrapassam a mera evocação de infomações semânticas acerca de si mesmo, pois o sujeito ao recordá-las tem consciência da vivência daquele evento como experiência própria, um estado mental que Tulving (1985) denominou de consciência autonoética, em oposição à consciência noética, característica da memória semântica e a anoética, própria da memória procedural.

$\mathrm{Na}$ perspectiva de Conway (2005), os aspectos diferenciadores entre a MA e a memória episódica são a autorreferência, a interpretação subjetiva do acontecimento recordado e a verificabilidade. Em comum, os dois sistemas de memória compartilham a mobilização dos atributos perceptivos e sensoriais associados às dimensões de espaço-tempo e a imagética, o que as distingue definitivamente da memória semântica.

Outro aspecto salutar para a discussão ora avançada diz respeito ao papel do componente emocional na organização da MA. Podem-se distinguir nos estudos duas posições extremas: a primeira defende que a emoção é a dimensão primária sobre a qual a MA é organizada; alternativamente, a posição do outro extremo argumenta que embora a emoção empreste um colorido à experiência autobiográfica, ela não pode ser considerada como princípio organizador da memória humana. Na perspectiva de Thompson e colaboradores (1996) a determinação acerca dos eventos a serem recordados pelos sujeitos enquanto ocorrência autobiográfica é influenciada pela avaliação emocional dos mesmos. Nesse sentido, identifica-se a primazia na evocação dos acontecimentos avaliados como extremos, produtores de emoções agradáveis, quando comparados com eventos considerados neutros ou desagradáveis pelos sujeitos. Os autores ainda destacam a interferência de outros fatores sobre o processo de recordação, tais como a frequência de ensaio; as expectativas associadas ao ato de recordar; a elaboração e participação ativa do sujeito no processamento da informação; e o estado de humor no qual o sujeito se encontra no momento da recordação (Gonçalves, 2007).

No que se refere à organização e funcionamento da MA diferentes estudos propõem uma hierarquia e funcionamento através de estruturas de conhecimento em rede. Anderson e Conway (1993) sugerem a existência de três níveis de conhecimento inter-relacionados, a saber, o nível geral, composto por conhecimentos abstratos relacionados a períodos da vida pessoal (por exemplo: "Quando morei em Paris"), o nível de conhecimento intermediário, composto por eventos gerais ou estendidos no tempo (por exemplo: “As minhas férias de verão quando morei em Paris em 2010”), por fim, o nível de conhecimento de eventos específicos ocorridos em local e tempo bem definidos (por exemplo: "Jantar com minha família no restaurante 'La Gourmandise' no dia 30 de abril de 2010 durantes as minhas férias de verão quando morei em Paris"). Por sua vez, Williams e Broadbent (1986) propuseram organização hierárquica para a MA a partir elaboração dos conceitos de MA especifica (acontecimentos ocorridos em local e tempo concretos, com duração máxima de um dia) e MA generalizada (eventos não circunscritos ou com duração superior a um dia).

As teorizações em torno da MA destacam quatro funções especificas para este sistema mnêmico: diretiva, social, autorrepresentativa (Bluck, Alea, Haberman, \& Rubin, 2005), às quais se acrescenta posteriormente a função adaptativa (Williams, Conway, \& Cohen, 2008). A função diretiva da MA refere-se à habilidade para a utilização de experiências do passado enquanto referência para a resolução de problemas no presente e como guia para ações futuras. Por sua vez, a função social está diretamente envolvida com o desenvolvimento, a manutenção e o enriquecimento das redes sociais, estabelecidas através de trocas de experiências pessoais com outros. A função autorrepresentativa ilustra o potencial das memórias pessoais para criar e manter coerentemente uma identidade ao longo do tempo. Por fim, a função de adaptação reflete o quanto tal sistema pode ser mobilizado para manter ou alterar padrões de humor desejáveis e indesejáveis.

A perda da capacidade de armazenar nova informação, ou de evocar experiências previamente armazenadas, implica em prejuízo de uma das principais funções da memória, ou seja, a construção e manutenção do senso de identidade (self), constituindo-se, portanto, em um dos declínios mais trágicos decorrentes da DA (Squire \& Kandel, 2003). De acordo com a compreensão do presente trabalho, a memória é um processo cognitivo complexo que possibilita ao sujeito o armazenamento de informações, abarcando conjunto de habilidades que permite, no caso da memória autobiográfica, a construção de uma história pessoal e de uma identidade (self) caracterizadas simultaneamente pela possibilidade de mudança e de estabilidade ao longo da vida.

Buscando contribuir para a elucidação acerca do curso 
evolutivo do declínio da MA nos pacientes com DA, assim como fornecer informações acerca das características da recordação autobiográfica nesta população, o objetivo do presente estudo foi a investigação da MA em idosos com DA nas fases leve e moderada, notadamente em termos de eventual diferenciação dos grupos no que se refere à especificidade e intensidade vivencial da recordação.

\section{Método}

\section{Participantes}

Participaram 44 idosos (com idades acima de 60 anos, conforme indicação da Organização Mundial de Saúde - WHO, 2011) de ambos os sexos, divididos em três grupos, DA leve ( $n$ $=15)$, DA moderada $(n=15)$, e Controle $(n=14)$, de acordo com o critério de presença e ausência do diagnóstico de DA e o estadiamento da demência, conforme ilustra a Tabela 1. Os idosos que compuseram os grupos DA leve e DA moderada eram pacientes acompanhados em Centro de referência municipal no diagnóstico, acompanhamento e tratamento de pacientes com DA, e os do grupo Controle, integrantes de programa social destinado ao público idoso, ambos localizados na cidade Natal, estado do Rio Grande do Norte.
Os critérios de inclusão foram: idade acima de 60 anos, ausência de diagnóstico de ansiedade e/ou depressão severas, ausência de alteraçãoes visuais e/ou auditivas não-corrigidas. Os critérios de exclusão foram a inadequação do sujeito a alguma das condições apontadas nos critérios de inclusão.

Todos os participantes e seus cuidadores assinaram o Termo de Consentimento Livre e Esclarecido (TCLE) e o estudo obteve aprovação do Comitê de Ética do Hospital Universitário Onofre Lopes- HUOL/UFRN.

\section{Instrumentos}

Constituição dos Grupos. Os grupos DA leve e DA moderada foram constituídos a partir da indicação feita pelos psiquiatras que integram a equipe de diagnóstico do CEASI (Centro Especializado de Assistência a Saúde do Idoso). Vale salientar que no referido serviço, funciona o programa de referência do município em diagnóstico e tratamento da DA, contando com equipe interdisciplinar especializada e que, por sua vez, se baseia nos critérios de DA provável, do National Institute of Neurological Comunicative Disorders and StrokeAlzheimer's Disease and Related Disorders (NINCDS-ADRDA).

O principal instrumento de diagnóstico clínico utilizado no CEASI é a Escala Clínica de Demência - CDR (desenvolvido por Hughes et al., 1992 e adaptado por Morris, 1993) que possibilita

Tabela 1

Distribuição de frequência dos grupos Controle, DA Leve, DA Moderada em função das variáveis Sexo, Idade e Escolaridade

\begin{tabular}{clccc}
\hline \multirow{2}{*}{ Sexo } & Masculino & $\begin{array}{c}\text { Grupo Controle } \\
(n=14)\end{array}$ & $\begin{array}{c}\text { DA leve } \\
(n=15)\end{array}$ & $\begin{array}{c}\text { DA moderada } \\
(n=15)\end{array}$ \\
& Feminino & 1 & 6 & 4 \\
Idade & Média $(D P)$ & 13 & 9 & 11 \\
& $60-69$ & $74,29 \pm 6,36$ & $79,0 \pm 7,07$ & $82,07 \pm 4,60$ \\
& $70-79$ & 5 & 2 & 0 \\
& $80-89$ & 6 & 4 & 2 \\
Escolaridade & Ens. Fund. Incompl. & 3 & 9 & 13 \\
& Ens. Fund. Compl. & 5 & 8 & 3 \\
& Ensino Méd. Compl. & 2 & 3 & 5 \\
& Ens. Sup. Compl. & 4 & 2 & 3 \\
\hline
\end{tabular}

classificar a prevalência dos diversos graus de demência. A escala investiga aspectos cognitivos e comportamentais, além de considerar as perdas cognitivas que interferem na realização das atividades de vida diária. $\mathrm{O}$ instrumento é constituído por seis categorias: memória, orientação, julgamento ou solução de problemas, relações comunitárias, atividades no lar ou de lazer e cuidados pessoais. Cada uma dessas seis categorias deve ser classificada em: 0 (nenhuma alteração); 0,5 (questionável); 1 (demência leve); 2 (demência moderada); e 3 (demência grave), exceto a categoria cuidados pessoais, que não tem o nível 0,5 . A categoria memória é considerada principal, ou seja, com maior significado e as demais categorias são secundárias. A classificação final do estadiamento da DA é obtida pela análise dessas classificações por categorias, seguindo um conjunto de regras elaboradas e validadas (Montaño \& Ramos, 2005). A Tabela 2 apresenta as principais características cognitivas e comportamentais dos quadros de DA Leve e Moderada de acordo a CDR (Bertolucci et al., 2005).

Avaliação da Memória Autobiográfica. O Teste de Memória Autobiográfica (TMA), desenvolvido por Pergher \& Stein (2008), consiste da apresentação, visual ou oral, de 15 palavras-pista com diferentes valências, sendo cinco positivas (elogio, agradável, diversão, animado e honesto), cinco negativas (trágico, infeliz, miséria, raivoso e decepcionado), e cinco neutras (fundo, embrulhado, vida selvagem, manualmente e alto). O sujeito é solicitado a recuperar lembranças autobiográficas específicas para cada palavra oferecida.

As respostas dadas para cada palavra-estímulo são codificadas em quatro categorias (Pergher \& Stein, 2008; Gonçalvez, 2007): 1) Não-memórias ou Associados Semânticos (quando o participante não consegue recuperar nenhuma lembrança ou fornece resposta que não se constitui em recordação autobiográfica); 2) Memórias Categóricas (refere-se a eventos repetidos, sem qualquer referência a um tempo específico); 3 ) 


\begin{tabular}{|c|c|c|}
\hline & $\begin{array}{c}\text { CDR } 1 \\
\text { DA Leve }\end{array}$ & $\begin{array}{c}\text { CDR } 2 \\
\text { DA Moderada }\end{array}$ \\
\hline Memória & $\begin{array}{l}\text { Perda de memória moderada, mais para } \\
\text { eventos recentes, atrapalha as atividades de } \\
\text { vida diária. }\end{array}$ & $\begin{array}{l}\text { Perda grave de memória, apenas assunto } \\
\text { altamente aprendido é recordado. }\end{array}$ \\
\hline Orientação & $\begin{array}{l}\text { Dificuldade moderada com relação ao } \\
\text { tempo, orientado em áreas familiares. } \\
\text { Necessita de ajuda }\end{array}$ & $\begin{array}{l}\text { Dificuldade grave com relação ao tempo, } \\
\text { desorientado quase sempre no espaço. }\end{array}$ \\
\hline $\begin{array}{l}\text { Julgamento e } \\
\text { resolução } \\
\text { de problemas }\end{array}$ & $\begin{array}{l}\text { Dificuldade moderada em lidar com } \\
\text { problemas, similaridades e diferenças; } \\
\text { julgamento social mantido. }\end{array}$ & $\begin{array}{l}\text { Dificuldade séria em lidar com problemas, } \\
\text { similaridades e diferenças; julgamento } \\
\text { social danificado. }\end{array}$ \\
\hline $\begin{array}{l}\text { Assuntos } \\
\text { Comunitários }\end{array}$ & $\begin{array}{l}\text { Não é independente nestas atividades, } \\
\text { parece normal em uma inspeção casual. }\end{array}$ & $\begin{array}{l}\text { Não há independência fora de casa, parece } \\
\text { bem o bastante para ser levado fora de casa. }\end{array}$ \\
\hline $\begin{array}{l}\text { Atividades } \\
\text { domésticas e } \\
\text { passatempo }\end{array}$ & $\begin{array}{l}\text { Prejuízo suave em tarefas em casa, tarefas } \\
\text { mais difíceis, passatempo e interesses } \\
\text { abandonados. }\end{array}$ & $\begin{array}{l}\text { Apenas tarefas simples são preservadas, } \\
\text { interesses muito restritos e pouco mantidos. }\end{array}$ \\
\hline Cuidado Pessoal & Necessita de ajuda. & Requer assistência ao vestir-se, para higiene \\
\hline
\end{tabular}

Memória Estendida (a lembrança remete a determinado período de tempo, com início, e fim determinados, e com duração superior a um dia); 4) Memória Específica (lembranças que tenha ocorrido num local determinado e que possuem uma localização temporal específica, com duração de um dia, ainda que o sujeito não se recorde quando).

Para a realização do presente estudo, a forma original do teste sofreu alteração, uma vez que durante o estudo-piloto os idosos apresentaram dificuldades na realização do TMA, notadamente associada à extensão do teste. Objetivando-se minimizar tal efeito, realizou-se estudo que possibilitou a redução do total de 15 palavras para um efetivo de seis palavras, sendo duas de valência positiva, duas de valência negativa e duas consideradas neutras. A partir da indicação dos idosos das palavras mais facilmente compreendidas dentre as quinze que compõem a versão original do instrumento, foram obtidas as 6 (seis) palavras que constituíram a versão do TMA utilizada. São elas: Valência Neutra: Novo e Infantil, Valência Negativa: Infeliz e Decepcionado; Valência Positiva: Elogio e Honesto

Questionário de Memória Autobiográfica. (QMA) trata-se de instrumento que avalia características de memórias produzidas a partir de palavras-estímulo. O diferencial de tal método é o fato de que o próprio indivíduo avalia e qualifica a memória recordada.

O QMA consiste de conjunto variável de itens na forma de afirmativas, avaliadas através de escala likert de sete pontos. Tal instrumento tem como objetivo investigar diferentes características da MA (Gauer \& Gomes, 2008): vivacidade da memória; presença de detalhes dos acontecimentos originais; importância pessoal atribuída ao evento; caráter incomum do evento; intensidade emocional do evento; conseqüências para a sua própria vida atribuídas ao evento pelo sujeito; freqüência de ensaio em pensamento e conversação.

\section{Procedimento}

A constituição dos grupos de idosos com DA nas fases leve e moderada foi realizada junto ao Programa de Alzheimer do CEASI (Centro Especializado de Assistência à Saúde do Idoso), localizado na cidade do Natal-RN. A seleção prévia dos idosos foi feita com base na análise dos prontuários. Posteriormente os pacientes diagnosticados com DA, bem como seus responsáveis, foram abordados com o objetivo de esclarecer a pesquisa e sondar acerca da disponibilidade e interesse da participação no estudo. Diante do aceite, os mesmos assinaram o TCLE e responderam às tarefas do MEEM e da Escala de Depressão Geriátrica (GDS). Desta forma, os idosos foram pré-selecionados de acordo com os critérios de inclusão e exclusão previamente estabelecidos, sendo posteriormente submetidos à avaliação da MA.

Os instrumentos que integraram o presente estudo foram aplicados individualmente, em sala apropriada do CEASI, nos horários da manhã e tarde, em período máximo de trinta dias após a entrevista de seleção. As instruções dos testes e subtestes foram padronizadas e lidas pelos examinadores, de modo a garantir a uniformidade na apresentação das tarefas.

O grupo controle foi composto por idosos que frequentavam o "Programa Saúde e Cidadania na Melhor Idade", na cidade do Natal-RN, considerando-se os critérios de inclusão e exclusão, bem como as variáveis sociodemográficas dos sujeitos dos outros grupos.

A aplicação do TMA seguiu as instruções propostas por Pergher e Stein (2008), descritas anteriormente. Inicialmente, foi apresentada uma palavra de valência neutra, seguida de palavras com valência positiva e negativa, sucessivamente. No entanto, quando o idoso conseguiu recordar "Memórias Específicas", foi aplicado o QMA, com o objetivo de investigar as qualidades fenomenais daquelas recordações. Vale ressaltar que não se aplicou o QMA para lembranças categorizadas como "Associados Semânticos", "Memórias Categóricas" ou "Memórias estendidas", uma vez que o QMA analisa exclusivamente a capacidade de recordar eventos pessoais específicos, explorando a vivacidade dessas memórias (Gauer \& Gomes, 2008). O procedimento de aplicação do TMA seguido do QMA foi repetido todas as vezes que o sujeito conseguiu evocar uma lembrança específica para as 6 palavras-estímulos apresentadas. Tal etapa de avaliação foi gravada em aparelho digital MP3.

Salienta-se que houve a necessidade de adaptação da escala likert proposta na versão original do instrumento, uma vez que as sutilezas de diferenciação dos níveis de intensidade vivencial da recordação não foram bem compreendidas pelos idosos. Nesse sentido, foi necessário realizar uma simplificação da escala que passou a ter três pontos: muito intenso (como se tivesse acabado de acontecer), pouco intenso (nem um pouco) ou com intensidade 
intermediária (mais ou menos).

Para a análise dos resultados obtidos nos instrumentos de investigação da memória autobiográfica (TMA e QMA) foi realizada Análise Descritiva Multidimensional para extração de estrutura de grupos (Two-Step Cluster Analysis), realizada em ambiente de tratamento informatizado de dados.

\section{Resultados}

O primeiro estágio de análise dos dados consistiu no estabelecimento de cruzamento entre as variáveis estatísticas (grupos e categorias das respostas), quanto à especificidade da MA (Memória Específica ou Memória Estendida), a partir das respostas evocadas pelas palavras-estímulo do TMA.

Observa-se na Figura 1 que a maioria das respostas produzidas no TMA pelo grupo Controle (52 respostas) foi categorizada nos níveis mais específicos da MA, ou seja, foram produzidas 33 respostas categorizadas como Memória estendida e 19 respostas categorizadas como Memória específica. Destacase que o grupo Controle produziu o menor número de Associados semânticos (nove AS) dentre os três grupos.

Após a realização da análise das frequências das respostas dos indivíduos quanto à especificidade, realizou-se análise multidimensional descritiva com o objetivo de detectar a existência de estrutura de grupos de indivíduos compostos por eventuais traços comuns em relação às variáveis estudadas (análise de grupos ou clusters). A princípio tal análise sugeriu a partição dos idosos em dois grandes grupos, sem exclusão de sujeitos: o Cluster 1, composto por 25 indivíduos e o Cluster 2 composto por 19 indivíduos.

De acordo com esta análise, a variável Grupo (DA leve, DA moderada e Controle) teve contribuição significativa $(p<0,05)$ na partição dos devidos Clusters. Nesse sentido, verifica-se que o Cluster $1(\mathrm{n}=25)$ é composto principalmente por sujeitos

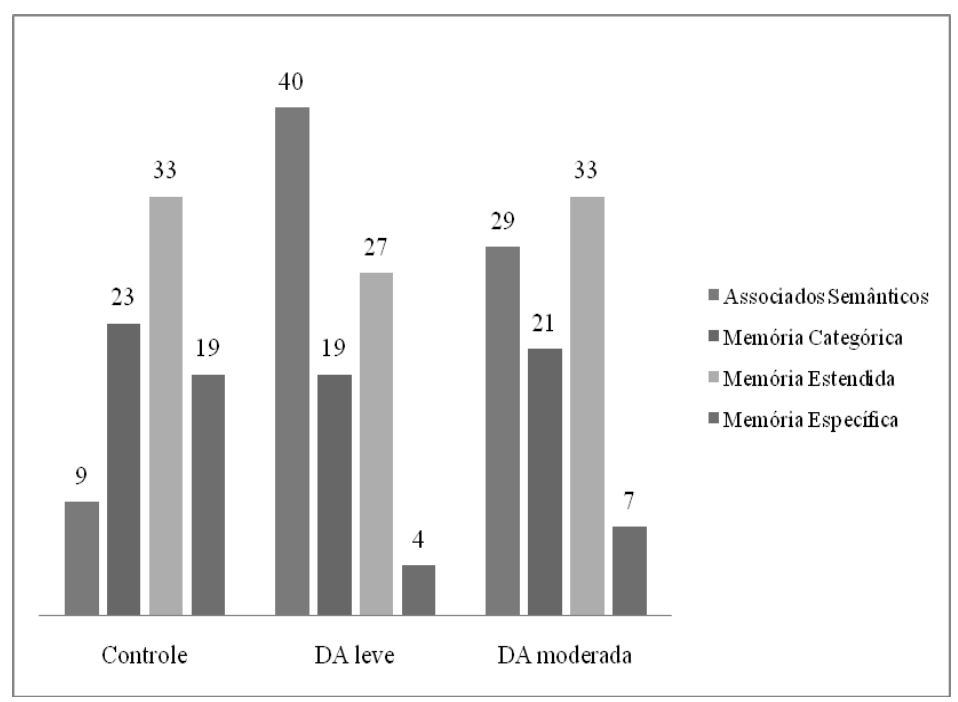

Figura 1. Frequência das respostas quanto ao nível de especificidade da MA para os grupos Controle, DA leve e DA moderada.

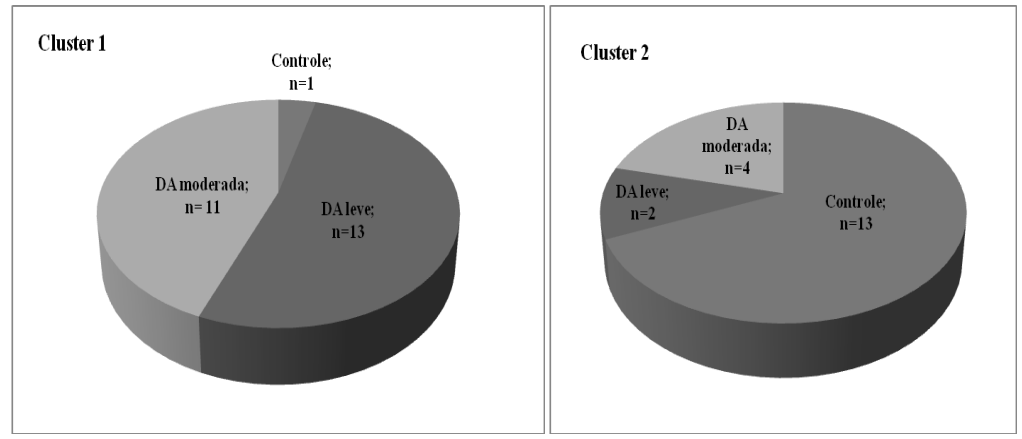

Figura 2. Composição dos Clusters 1 e 2 quanto ao grupo de origem dos sujeitos (Controle, DA leve e DA moderada).

diagnosticados com DA na fase leve $(n=13)$ e com DA na fase moderada $(n=11)$, sendo apenas um sujeito oriundo do grupo Controle. Por sua vez, o Cluster $2(n=19)$ foi formado basicamente pelos indivíduos do grupo Controle $(n=13)$ e alguns indivíduos dos grupos DA leve $(n=2)$ e DA moderada $(n=4)$.

A categoria "Associados Semânticos", produção de evocações no nível mais generalizado de respostas para o TMA, também mostrou-se igualmente significativa na diferenciação dos grupos, sendo portanto, uma variável de contribuição relevante para a formação do Cluster 2

A análise das características dos Clusters quanto à frequência de produção das respostas categorizadas como "Associados Semânticos", indica que o Cluster 1 foi constituído por aqueles indivíduos que apresentaram elevada frequência dessa categoria 


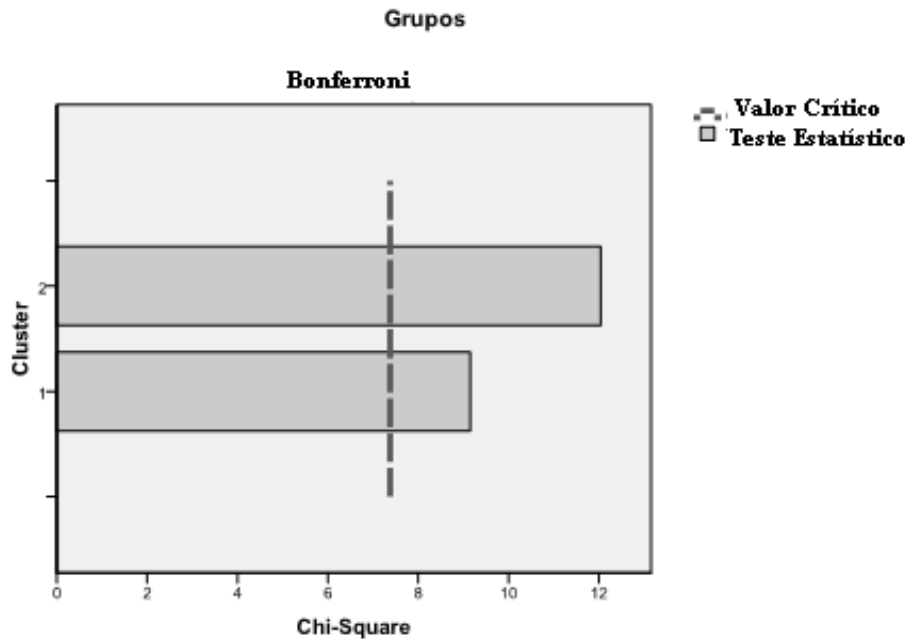

Figura 3. Níveis de contribuição (em métrica do qui-quadrado) das categorias da variável "Grupos" para a constituição dos clusters 1 e 2 (valor crítico para significância em termos de $5 \%$ representados ela linha pontilhada vertical).

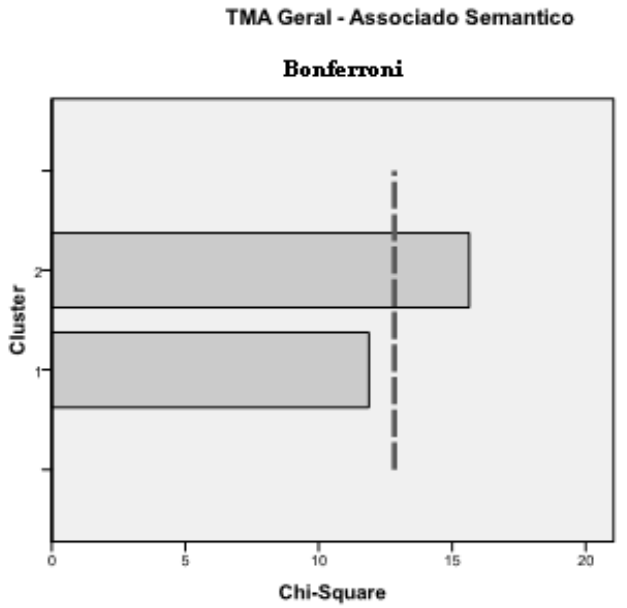

$$
\begin{aligned}
& \text { Valor Crítico } \\
& \text { Teste Estatistico }
\end{aligned}
$$

Figura 4. Níveis de contribuição (em métrica do qui-quadrado) das categorias da variável "Associados Semânticos" para a constituição dos clusters 1 e 2 (valor crítico para significância em termos de 5\% representados ela linha pontilhada vertical).

de respostas, sendo portanto, sujeitos que apresentaram dificuldades para evocar recordação autobiográfica específicas. Por sua vez, o Cluster 2 foi constituído por indivíduos que apresentaram menor freqüência de resposta categorizada como “Associado Semântico", logo, indivíduos que produziram grande número de recordações acompanhadas de vivência emocional.

Ainda de acordo com a análise de Cluster realizada, não houve outra categoria do TMA (Memória Categórica, Memória Estendida ou Memória Específica) com contribuição estatisticamente relevante para as partições encontradas. Dessa forma, além do diagnóstico de DA, apenas a capacidade ou não de evocar uma recordação autobiográfica (categórica, estendida ou específica) foi determinante na diferenciação entre os indivíduos dos dois Clusters encontrados.

Por fim, foi realizada a análise dos dados concernentes à aplicação do QMA sobre o conjunto de 30 "Memórias
Específicas" produzidas no TMA pelo total de participantes (Grupo Controle $=19$, DA Moderada $=7$ e DA Leve $=4)$. Observa-se que para o grupo Controle, dentre as variáveis que caracterizam a MA, aquelas que mais contribuíram com a experiência da recordação fenomenal foram: "eu sinto como se estivesse revivendo o acontecimento original" (18 vezes; 94,7\%); "eu posso de fato lembrar dele, ao invés de apenas saber que aconteceu" (17 vezes; 89,4\%); "Eu acredito que o episódio em minha memória realmente ocorreu da forma como eu lembro, ou seja, eu não imaginei ou fabriquei algo que não aconteceu" (18 vezes, 94,7\%); "ele me vem, em palavras ou imagens, como uma história coerente, e não como uma cena, observação ou fato isolado" (18 vezes; 94,7\%); e "Desde que aconteceu, eu pensei sobre este episódio" (18 vezes). Por outro lado, as variáveis características que foram referidas com menor frequência pelo grupo Controle foram: "eu ou outras pessoas estamos falando." 
(13 vezes; 68,4\%); "eu posso sentir agora as emoções que eu senti naquela hora" (12 vezes; $63,2 \%$ ); "ele me vem em palavras" (13 vezes; 68,4\%); e "Este episódio teve consequências importantes para mim" (11 vezes; $57,9 \%$ ).

Por sua vez, para os sujeitos do grupo DA leve, nove características $(50 \%)$ foram referidas como sendo de grande intensidade vivencial no momento da recordação ("eu posso lembrar o cenário onde ocorreu"; "ele me vem, em palavras ou imagens, como uma história coerente, e não como uma cena, observação ou fato isolado"; "Esta memória é significante para minha vida"; "Eu acredito que o episódio em minha memória realmente ocorreu da forma como eu lembro, ou seja, eu não imaginei ou fabriquei algo que não aconteceu"; "Desde que aconteceu, eu pensei sobre este episódio"; "Desde que aconteceu, eu conversei com alguém sobre este episódio"; "Este episódio teve grande intensidade emocional" e; "Este episódio teve consequências importantes para mim".

Finalmente, constatou-se que para o grupo DA moderada não houve nenhum item do QMA referido com sendo de alta intensidade vivencial. No entanto, destaca-se que aquelas que apareceram com maior frequência foram: "eu posso vê-lo na minha mente"(5 vezes; 71,4\%); "eu sei como os elementos estavam distribuídos no espaço" (seis vezes; 85,7\%); "eu posso de fato lembrar dele, ao invés de apenas saber que aconteceu" (seis vezes, 85,7\%); e "Eu acredito que o episódio em minha memória realmente ocorreu da forma como eu lembro, ou seja, eu não imaginei ou fabriquei algo que não aconteceu" (5 vezes, $71,4 \%)$.

\section{Discussão}

A MA é o aspecto da memória que está relacionado à recordação de eventos passados experimentados pessoalmente (Conway \& Pleydell-Pearce, 2000). Sabe-se que tal sistema de memória é diretamente afetado pelo processo de envelhecimento (Nyberg et al., 2003). Nesse sentido, sugere-se que o envelhecimento esteja associado à diminuição da capacidade de recordar detalhes sensório-perceptivos, afetivos ou espaçotemporais específicos, o que pode comprometer a experiência de associação do evento em questão à sua própria pessoa. (Piolino, Desgranges, Benali, \& Eustache, 2002; Piolino et al., 2006).

A experiência de recordação autobiográfica apresenta comprometimento junto a grupos clínicos específicos, tais como o autismo (Bruck, London, Landa, \& Goodman, 2007), patologias do lobo frontal (Della Sala, Laiacona, Spinnler, \& Trivelli,1993), esquizofrenias (Corcoran \& Frith, 2003), depressão (Gonçalves, 2007; Pergher \& Stein, 2008) e demências semântica e frontotemporal (Ivanoiu, Cooper, Shanks, \& Venneri, 2006). Tais afecções apresentam alterações em componentes psicológicos relacionados à recordação de eventos autobiográficos, acarretando perturbações graves para a manutenção do núcleo identitário do indivíduo, com desdobramentos igualmente graves na área da interação sócioafetiva (Hazin, Lemos, \& Da Rocha Falcão, 2012)

Buscando aprofundar as relações entre MA e grupos clínicos específicos, a presente pesquisa investigou a MA em idosos em três contextos, a saber, na ausência de afecções neuropsiquiátricas, em idosos diagnosticados com DA na fase leve e em idosos com DA na fase moderada. Tal investigação centrou-se em eventuais aspectos diferenciadores dos grupos, notadamente no que se refere à especificidade e à intensidade vivencial da recordação, conforme discutido nas subseções seguintes.

\section{Especificidade das memórias autobiográficas}

No tocante ao aspecto especificidade da recordação pode-se concluir que o diagnóstico de DA está associado à diminuição da capacidade de especificar recordações autobiográficas, fenômeno denominado de supergeneralização. A supergeneralização referese à incapacidade de circunscrever um único evento no tempo e no espaço, quando o sujeito é apresentado a uma palavra-estímulo. É interessante notar que o fenômeno da supergeneralização foi inicialmente identificado em indivíduos com diagnóstico de depressão (Pergher \& Stein, 2008). Autores sugerem que tal constatação estaria associada a declínio cognitivo, notadamente, em termos da capacidade de inibir pensamentos distratores e da diminuição dos recursos atencionais (Robertson, Myerson, \& Hale, 2006).

Observou-se igualmente a tendência dos indivíduos com DA a relatar memórias em período de tempo estendido ou através de memórias categóricas. Nessa direção, Williams (1996), discute que a tentativa de evocação de um evento específico, exige primeiramente a capacidade de descrição geral do evento (nível mais elevado da hierarquia das memórias), e posteriormente a busca por acesso a eventos específicos. Em alguns casos, tal busca é interrompida, resultando em recordação final generalizada, o que denomina-se bloqueio mnemônico. De acordo com o autor, este bloqueio seria causado por uma capacidade cognitiva insuficiente para inibir o nível de descrição geral, e chegar a marcadores temporais e espaciais específicos.

Partindo da hipótese supracitada, Phillips e Williams (1997) concluíram que a recordação de aspectos específicos da MA diminui com o aumento dos prejuízos cognitivos em adultos. Examinaram amostra heterogênea de pacientes idosos com variados tipos de patologias e concluíram que pacientes saudáveis não apresentavam supergeneralização da MA, ao contrário dos grupos clínicos.

De forma semelhante ao encontrado no estudo acima citado, Donix e colaboradores (2010) enfatizaram igualmente o impacto da DA sobre o processamento cognitivo e a qualidade da recuperação autobiográfica em geral. Seus dados evidenciaram que os pacientes com DA, já em fase muito precoce da doença, reportam significativamente mais "Memórias Categóricas" e "Memórias Estendidas", e significativamente menos respostas específicas, quando comparados a sujeitos controle com a mesma idade, independente da valência da palavra-estímulo. Este estudo também sugere uma relação entre a preservação da especificidade da MA e a disponibilidade de recursos cognitivos dos sujeitos.

Na mesma direção, o estudo de Greene, Hodges e Baddeley (1995), realizado com pacientes com DA leve e levíssima (com pontuação no MEEM >24), verificou prejuízos na MA desses sujeitos em relação aos controles. Dessa forma, assim como no presente estudo, a MA encontra-se prejudicada em fase muito inicial da doença, confrontando a ideia de que a memória remota 
estaria inicialmente preservada em todos os seus aspectos. Tal achado leva à hipótese de que outras áreas além do hipocampo estão envolvidas na recuperação da MA (Greene, Hodges, \& Baddeley, 1995).

Estudos diversos ressaltam que as MAs são construídas a partir de vários tipos de conhecimento, que vão do geral para o específico, através de processos generativos. (Anderson \& Conway, 1993; Conway \& Pleydell-Pearce, 2000). Estes processos estão relacionados com o sistema frontal/executivo, que inclui processamento de estratégias, de elaboração, avaliação e inibição, intimamente ligado a outras propostas relativas ao controle do processamento (Burgess \& Shallice, 1996; Moscovitch \& Melo, 1997, Norman \& Shallice, 1986). Os autores citados observaram que a MA e as FE declinam conforme a evolução da DA (Greene, Hodges, \& Baddeley, 1995). As funções executivas envolvidas no processo de planejamento, controle e avaliação, são referenciadas em alguns estudos como sendo prejudicadas desde os estágios iniciais da doença de Alzheimer (Greene, Hodges, \& Baddeley, 1995). Dessa forma, chega-se à hipótese de que o lobo frontal, região cerebral associada às funções executivas, é vital para a recuperação, reconstrução e verificação da MA (Hodges \& McCarthy, 1993).

Ainda nessa direção, Sebastian, Menor e Elosua (2001), constataram que sujeitos com DA apresentam tendência à perseveração. Os autores sugerem que tais déficits estão associados à dificuldade de alternância do foco atencional, ou seja, na passagem do foco de um estímulo ou evento para outro. Pode-se dizer que a atenção permanece "ancorada" no item anteriormente recordado. Estas perseverações têm sido encontradas em pacientes com disfunções executivas indicando déficit de "supervisão" ou "monitoramento" da execução da ação (Stuss \& Alexander, 2005).

Os resultados dos estudos supracitados são corroborados pelos resultados obtidos pela presente pesquisa, notadamente em termos da natureza perseverativa de respostas no TMA. Observou-se que apenas um dos idosos do grupo Controle apresentou perseveração no desempenho do TMA. No entanto, sete sujeitos com DA leve $(n=15)$ e nove sujeitos com DA moderada $(n=15)$ apresentaram, pelo menos, 3 evocações semelhantes (descrição de um mesmo episódio) para diferentes palavras do TMA (Sandson \& Albert, 1984).

Sendo assim, os dados acima expostos reforçam a associação entre a incapacidade de recordação de eventos específicos e o declínio cognitivo, notadamente em termos de recursos atencionais e funcionamento executivo. Tais evidências são de grande relevância para a maior compreensão do processo de declínio da MA, de modo a ofertar subsídios para práticas de reabilitação que contribuam para maior qualidade de vida desse grupo. Isso porque a especificidade da MA interfere no desempenho na solução de problemas sociais e serve como função de orientação, direcionando o comportamento social por toda a vida (Piolino, Desgranges, \& Eustache, 2009).

\section{Intensidade vivencial das memórias autobiográficas}

Talarico, Labar e Rubin (2004) discutem que a intensidade fenomenal da recordação é a principal propriedade da MA, mais do que a valência ou a idade do evento em questão. Quanto às características fenomenais que acompanham a experiência consciente ao evocar uma MA (relacionadas à consciência autonoética), investigadas através da aplicação do QMA, QMA, a análise específica das produções dos três grupos revelou que o grupo Controle apresentou elevada intensidade vivencial para todas as recordações específicas produzidas.

No grupo DA Leve, foram produzidas apenas quatro memórias específicas, sendo duas delas associadas à elevada intensidade vivencial em todos os itens do QMA. Por fim, a investigação da produção do grupo com DA moderada sugeriu que, embora tenha evocado maior número de "Memórias específicas" comparado ao grupo DA Leve, o grupo DA Moderada apresentou menor frequência de respostas associadas à elevada intensidade vivencial.

De forma geral, os itens associados às recordações foram classificados como possuindo média ou nenhuma intensidade vivencial. Tal aspecto pode estar associado à perda do caráter emocional associado à recordação, ou seja, os indivíduos conseguem reconhecer que os eventos recordados aconteceram com eles próprios, mas não reeditam a experiência emocional atrelada a tais eventos.

\section{Considerações Finais}

As reflexões avançadas a partir deste estudo sugerem que o declínio da memória autobiográfica nos quadros de Demência de Alzheimer parece iniciar-se a partir dos aspectos emocionais da MA, ficando preservados, em fases intermediárias da doença apenas aqueles associados aos fatos autobiográficos e ao esquema de si. Os sujeitos do grupo DA Moderada recordam os eventos autobiográficos desconectados de seu caráter emocional, reconhecendo-os como pessoais apenas pela certeza de que estes aconteceram com eles próprios e a partir de alguns detalhes. Por sua vez, o grupo DA Leve mantém a experiência vivencial associada a aspectos emocionais. No entanto, nota-se que a frequência de tais produções é inferior a do grupo Controle, sugerindo dificuldades maiores na recuperação de eventos autobiográficos específicos. Os achados da presente pesquisa resgatam a indissociabilidade das dimensões cognitivas e afetivas no funcionamento psicológico humano. Nesse sentido, o declínio do sistema da MA em idosos com DA revela o impacto de tal dissociação em contextos patológicos.

Por fim, aponta-se como principal limitação da presente pesquisa, encoranjando-se a realização de estudos futuros, a ausência de investigação do impacto das modificações realizadas na aplicação do QMA e do TMA. Apesar da flexibilidade na aplicação dos mesmos, suscitadas inclusive por seus propositores, os resultados ora discutidos são resultantes de contexto experimental que, embora semelhante para os três grupos investigados, não permite a discussão destes resultados com outros dados fomentados por estudos realizados no Brasil com os mesmos instrumentos.

\section{Referências}

Abreu, I. D., Forlenza, O. V., \& Barros, H. L. (2005). Demência de Alzheimer: 
correlação entre memória e autonomia. Revista de Psiquiatria Clínica, 32(3), 131-136.

American Psychiatric Association. (2002). Delirium, demência, transtornos amnéstico e outros transtornos cognitivos. In Manual Diagnóstico e Estatístico de Transtornos Mentais. DSM-IV (pp.121-60). Porto Alegre: Artes Médicas.

Anderson, A. J., \& Conway, M. A (1993). Investigating the structure of autobiographical memories. Journal of Experimental Psychology: Learning. Memory, and Cognition, 19(5), 1178-1196.

Bertolucci, P. H. F., Okamoto, I.H., Brucki, S. M., Siviero, M. O., Toniolo Neto, J., \& Ramos, L. R. (2005). Applicability of the CERAD neuropsychological battery to Brazilian elderly. Arquivos de Neuropsiquiatria, 59(3A):532-536.

Bluck, S., Alea, N., Habermas, T., \& Rubin, D. (2005). A tale of three functions: the self-reported uses of autobiographical memory. Social Cognition, 23(1), 91-117.

Bruck, M., London, K., Landa, R., \& Goodman, J. (2007). Autobiographical memory and suggestibility in children with autism spectrum disorder. Development and Psychopathology, 19, 73-95.

Burgess, P. W., \& Shallice, T. (1996). Response suppression, initiation, and strategy use following frontal lobe lesions. Neuropsychologia, 34(1), 263273.

Conway, M. A. (2005). Memory and the self. Journal of Memory and Language. $53,594-628$.

Conway, M. A., \& Pleydell-Pearce, C. W. (2000) The construction of autobiographical memories in the self memory system. Psychological Review, 107, 261-288.

Corcoran, R, \& Frith, R (2003). Autobiographical memory and theory of mind: evidence of a relationship in schizophrenia. Psychological Medicine, 33, 897-905.

Della Sala, S., Laiacona, M., Spinnler, H., \& Trivelli, C. (1993). Autobiographical recollection and frontal damage. Neurapsychologia 31, 823-839.

Donix, M. Brons, C., Jurjanz, L., Poettrich, K., Winiecki, P., \& Holthoff, V. A. (2010). Overgenerality of autobiographical memory in people with amnestic mild cognitive impairment and early Alzheimer's Disease. Archives of Clinical Neuropsychology 25, 22-27.

Gauer, G., \& Gomes, W. B. (2008). Recordação autobiográfica: reconsiderando dados fenomenais e correlatos neurais. Aletheia, 27(1), 36-50.

Gonçalves, D. A. C. S. (2007). Estimulação e promoção de memórias autobiográficas específicas como metodologia de diminuição de sintomatologia depressiva em pessoas idosas (Dissertação de Mestrado). Recuperado de http://hdl.handle.net/1822/6506 do Minho, Braga.

Greenberg, D. L., \& Rubin, D. C. (2003). The Neuropsychology of autobiographical memory. Cortex, 39, 687-728.

Greene, J. D. W., Hodges, J. R., \& Baddeley, A. D. (1995) Autobiographical memory and executive function in early dementia of Alzheimer type, Neuropsychologia, 33, 1647-1670.

Hazin, I., Lemos, C. A., \& Da Rocha Falcão, J. (2012). Evidências científicas e modelos conceituais da memória autobiográfica: subsídios para a reabilitação neuropsicológica. In J. Abrisqueta-Gomez (Org.), Reabilitação Neuropsicológica: metodologia científica e modelos conceituais na prática clinica (pp. 154-167). Porto Alegre: Artmed.

Herrera, E., Caramelli, P., Silveira, A. S., \& Nitrini, R. (2002). Epidemiologic survey of dementia in a community-dwelling Brazilian population. Alzheimer Disease \& Associated Disorders, 16, 103-108.

Hodges, J. R., \& McCarthy, R. A. (1993). Autobiographical amnesia resulting from bilateral paramedian thalamic infarction: a case study in cognitive neurobiology. Brain, 116, 921-940.

Hughes, C. P., Berg, L., Danziger, W. L., Coben, L. A., Martin, R. L. (1982). A new clinical scale for the staging of dementia. Brazilian Journal of Psychiatry, 140, 566-72.

Ivanoiu, A., Cooper, J. M., Shanks, M. F., \& Venneri, A. (2006). Patterns of impairment in autobiographical memory in the degenerative dementias constrain models of memory. Neuropsychologia, 44, 1936-1955.

Machado, J. C. B. (2002). Doença de Alzheimer. In E. V. Freitas, L. Py, F. A. X. Cançado, \& M. L. Gorzoni (Orgs.), Tratado de geriatria e gerontologia (pp. 262-280). Rio de Janeiro: Guanabara Koogan.

Morris, J. (1993) The Clinical dementia rating (CDR): current version and scoring rules. Neurology, 43(11), 2412-2414.

Moscovitch, M., \& Melo, B. (1997), Strategic retrieval and the frontal lobes: evidence from confabulation and amnesia. Neuropsychologia, 35, 1017-1034.

Norman, W, \& Shallice, T. (1986). Attention to action. In R. J. Davidson, G. E. Schwartz, \& D. Shapiro (Orgs.), Consciousness and self regulation: advances in research and theory (pp. 1-18). Nova Iorque: Plenum.

Nyberg, L., Maitland, S. B., Rönnlund, M., Bäckman, L., Dixon, R. A., Wahlin, A., \& Nilsson, L-G (2003). Selective adult age differences in an age-invariant multifactor model of declarative memory. Psychol Aging, 18, 149-160.

Pergher, G. K., \& Stein, L. M. (2008). Recuperando memórias autobiográficas: avaliação da versão brasileira do Teste de Memória Autobiográfica. PSICO, 39(3), 299-307.

Phillips, S., \& Williams, M. G. (1997). Cognitive impairment, depression and the specificity of autobiographical memory in the elderly. Journal of Clinical Psychology, 36, 341-347.

Piolino, P., Desgranges, B., Benali, K., \& Eustache, F. (2002). Episodic and semantic remote autobiographical memory in aging. Memory, 10, 239-257.

Piolino, P., Desgranges, B., \& Eustache, F. (2009). Episodic autobiographical memory over the course of time: cognitive, neuropsychological and neuroimaging findings. Neuropsychologia, 47(11), 2314-2329.

Piolino, P., Hannequin, D., Desgranges, B., Girard, C., Beaunieux, H., Giffard, B., ...\& Eustache, F. (2006). Right ventral frontal hypometabolism and abnormal sense of self in a case of disproportionate retrograde amnesia. Cognitive Neuropsychology, 22, 1005-1034.

Qiu, C., Kivipelto, M., \& Strauss, E. (2009). Epidemiology of Alzheimer's disease: occurrence, determinants, and strategies toward intervention. Dialogues in Clinical Neuroscience, 11(2), 111-128.

Robertson, S., Myerson, J., \& Hale, S. (2006). Are there age differences in intraindividual variability in working memory performance? Journal of Gerontology Psychological Sciences, 61B(1), 18-24.

Sandson, J., \& Albert, M. L. (1984). Varieties of perseveration. Neuropsychologia, $22,715-732$.

Scazufca, M., Menezes, P. R., Vallada, H. P., Crepaldi, A. L., Pastor-Valero, M., Coutinho, L. M. S., .. \& \& Almeida, O. P. (2008), High prevalence of dementia among older adults from poor socioeconomic backgrounds in São Paulo, Brazil. International Psychogeriatrics, 20(2), 394-405.

Sebastian, M. V., Menor, J., \& Elosua, R. (2001). Patterns of errors in short term forgetting in $\mathrm{AD}$ and ageing. Memory, 9, 223-231.

Squire, L. R., \& Kandel, E. R. (2003). A memória e as bases biológicas da individualidade. In L. R.Squire \& E. R. Kandel (Orgs.), Memória: da mente às moléculas (pp. 211-240). Ed. Artmed: Porto Alegre.

Stuss, D., \& Alexander, M.P. (2005). Does damage to the frontal lobes produce impairment in memory? Current Directions in Psychological Science, 14, 84-88.

Talarico, J. M., Labar, K. S., \& Rubin, D. C. (2004). Emotional intensity predicts autobiographical memory experience. Memory \& Cognition, 32(7), 1118 1132.

Tulving, E. (1985). How many memory systems are there? American Psychologist, 40, 385-398.

Vilela, L. P., \& Caramelli, P. (2006). A doença de Alzheimer na visão de familiares de pacientes. Revista da Associação Médica Brasileira, 52(3), 148-152.

Williams, H. L., Conway, M. A., \& Cohen, G. (2008). Autobiographical memory. In G. Cohen \& M. A. Conway (Orgs.), Memory in the real world (3rd Ed.) (pp. 21-90). Hove, UK: Psychology Press.

Williams, J. M., \& Broadbent, K. (1986). Autobiographical memory in suicide attempters. Journal of Abnormal Psychology, 95(2), 144-149.

World Health Organization (2011). World health statistics. Genebra: WHO Press. 
144 C. A. Lemos, I. Hazin \& J. T. R. Falcão

Caroline Araújo Lemos, mestre em Psicologia pela Universidade Federal do Rio Grande do Norte, é professora na Universidade Potiguar (UnP). Endereço: Av. Engenheiro Roberto Freire, 1684. Ponta Negra. Natal/RN. CEP: 59080-400. Telefone: (84) 3215.1234.E-mail: carolpsilemos@yahoo.com.br

Izabel Hazin, doutora em Psicologia Cognitiva pela Universidade Federal de Pernambuco, é professora Adjunta 3 na Universidade Federal do Rio Grande do Norte. E-mail: izabel.hazin@gmail.com

Jorge Tarcísio da Rocha Falcão, doutor em Psicologia da Aprendizagem e do Desenvolvimento pela Universitè Paris V, é professor Titular na Universidade Federal do Rio Grande do Norte. E-mail: falcao.jorge@gmail.com 